\title{
Quantized Feedback Control Design of Nonlinear Large-Scale Systems via Decentralized Adaptive Integral Sliding Mode Control
}

\author{
Yan-Mei Xue, ${ }^{1}$ Bo-Chao Zheng, ${ }^{2}$ and Dan Ye ${ }^{3}$ \\ ${ }^{1}$ School of Mathematics and Statistics, Nanjing University of Information Science and Technology, Nanjing, Jiangsu 210044, China \\ ${ }^{2}$ CICAEET, School of Information and Control, Nanjing University of Information Science and Technology, \\ Nanjing, Jiangsu 210044, China \\ ${ }^{3}$ College of Information Science and Engineering, Northeastern University, Shenyang, Liaoning 110819, China
}

Correspondence should be addressed to Bo-Chao Zheng; zhengbochao81@126.com

Received 14 April 2015; Revised 19 June 2015; Accepted 12 July 2015

Academic Editor: Sarah K. Spurgeon

Copyright (C) 2015 Yan-Mei Xue et al. This is an open access article distributed under the Creative Commons Attribution License, which permits unrestricted use, distribution, and reproduction in any medium, provided the original work is properly cited.

\begin{abstract}
A novel decentralized adaptive integral sliding mode control law is proposed for a class of nonlinear uncertain large-scale systems subject to quantization mismatch between quantizer sensitivity parameters. Firstly, by applying linear matrix inequality techniques, integral-type sliding surface functions are derived for ensuring the stability of the whole sliding mode dynamics and obtaining the prescribed bounded $\mathscr{L}_{2}$ gain performance. Secondly, the decentralized adaptive sliding mode control law is developed to ensure the reachability of the sliding manifolds in the presence of quantization mismatch, interconnected model uncertainties, and external disturbances. Finally, an example is shown to verify the validity of theoretical results.
\end{abstract}

\section{Introduction}

It is well known that quantization phenomena are frequently encountered in numerous practical engineering systems; as a result, the control design considering signal quantization has received much attention since 1998. Numerous well-known results have been issued, such as stability analysis of linear and nonlinear continuous-time systems $[1,2]$, distributed coordination of multiagent systems [3], event-triggered control, $H_{\infty}$ or stabilization for networked control systems [4-6], $H_{\infty}$ filter design for linear continuous-time systems [7], robust or fault-tolerant control using sliding mode control technique [8-12], and $\mathrm{H}_{2}$ control of continuous-time linear systems [13] subject to input quantization and matched external disturbances.

In this paper, the input quantization problem will be considered. Though similar discussions are also investigated in existing results, such as in [13-15], it should be pointed out that the research works there and in $[1,2,4,7,16]$ are all built on the assumption that quantizer sensitivity parameters, which are cultivated separately at the coder and decoder sides, are identical all the time or the ratio of them keeps unchanged [17-19]. This assumption actually requires that the adjustment synchronization of sensitivity parameters is enforced at every time step in practical engineering applications; thus it might not be implementably induced by hardware imperfections of coder and decoder. For coping with this problem, a timevarying ratio model with known lower and upper boundaries is first introduced in [20]. By utilizing robust control technique, the $\mathrm{H}_{2}$ control design for a class of uncertain systems subject to input quantization mismatch is investigated there.

On the other hand, the large-scale systems are widely used in modern industrial systems, and thus decentralized control design has been well studied in the past two decades; see [21-23] and the references therein. In [21, 22], based on sliding mode control technique, the decentralized output feedback of nonlinear interconnected systems is well investigated. Specially, since the large-scale systems usually are linked via networks, the decentralized control involving signal quantization has aroused the attention of scholars $[24,25]$. However, to the best of our knowledge, no results 
have considered the decentralized control design via sliding mode control technique for large-scale systems subject to input quantization mismatch.

Motivated by all the mentioned above, we will address the problems of quantized feedback decentralized adaptive integral sliding mode control design for a class of nonlinear uncertain large-scale systems with input quantization mismatch. The purpose is to contribute to the development of quantized feedback sliding mode control theory and the bounded $\mathscr{L}_{2}$ gain performance analysis for nonlinear largescale uncertain system. The main contribution includes two aspects. First, applying linear matrix inequality technique, sufficient conditions are derived for guaranteeing the robust stability of sliding mode dynamics with bounded $\mathscr{L}_{2}$ gain performance. Second, considering the established boundaryunknown time varying ratio relation model of the quantization sensitivity parameters, the decentralized adaptive integral sliding mode control strategy is proposed to eliminate the effect of the mismatch of the quantization sensitivity parameters, model uncertainties, and external disturbances.

This paper is organized as follows. Section 2 provides a system description, relation model for the mismatch of the quantization sensitivity parameters, and some preliminary results. The main results are presented in Section 3. In Section 4, an example is given to illustrate the results and this paper is concluded in Section 5 finally.

The following notations are used in this paper. $\mathbb{R}^{n}$ denotes the $n$-dimensional Euclidean space; $A^{T}$ denotes the transpose of matrix $A$; and $I$ and 0 represent the identity matrix and a zero matrix in appropriate dimension, respectively. $X>$ $0(X \geq 0)$ means that $X$ is real symmetric and positive definite (semipositive definite). The symbol $\mathrm{He}(X)$ represents $X+X^{T} \cdot|x|_{p}$ denotes the $p$-norm of the vector $x$; that is, $|x|_{p}=\left(\left|x_{1}\right|^{p}+\left|x_{2}\right|^{p}+\cdots+\left|x_{n}\right|^{p}\right)^{1 / p}, p \geq 1$. When $p=\infty$, $|x|_{\infty}=\max _{1 \leq i \leq n}\left|x_{i}\right|$. Specially, the notation $|\cdot|$ denotes the standard Euclidean norm of a vector, or the induced norm of a matrix, respectively. In symmetric block matrices, we use a notation $*$ to represent a term that is inferred by symmetry.

\section{Problem Statement and Preliminaries}

In this paper, the following class of nonlinear uncertain large-scale systems with $N$ interconnected subsystems is considered:

$$
\begin{aligned}
\dot{x}_{i}(t)= & \left(A_{i}+\Delta A_{i}(t)\right) x_{i}(t)+B_{i} Q_{i}\left(u_{i}(t)\right) \\
& +B_{i} f_{i}(t, x, p)+D_{i} \omega_{i}(t) \\
z_{i}(t)= & C_{i} x_{i}(t)+H_{i} \omega_{i}(t)
\end{aligned}
$$

where $x_{i} \in \mathbb{R}^{n_{i}}, u_{i} \in \mathbb{R}^{m_{i}}, Q_{i}\left(u_{i}\right) \in \mathbb{R}^{m_{i}}$, and $z_{i} \in$ $\mathbb{R}^{p_{i}}$ are the state, the control input, quantized control input, and controlled output of the $i$ th subsystem, respectively; $i=1,2, \ldots, N . \omega_{i} \in \mathbb{R}^{q_{i}}$, belonging to $\mathscr{L}_{2}[0, T]$, is the exogenous input of the $i$ th subsystem. $\Delta A_{i}(t) \mathbb{R}^{n_{i} \times n_{i}}$ is the unknown mismatched time varying model uncertainty in the $i$ th subsystem. $f_{i}(t, x, p) \in \mathbb{R}^{m_{i}}$ describes the nonlinear interconnected term affecting the $i$ th subsystem, where $x=\left[x_{1}^{T}, x_{2}^{T}, \ldots, x_{N}^{T}\right]^{T}$ and $p \in \mathbb{R}^{r}$ is uncertain system parameter. Some assumptions are made in the following.

Assumption 1. Time varying uncertainties $\Delta A_{i}(t)$ satisfy

$$
\begin{aligned}
\Delta A_{i}(t) & =F_{i} \Delta_{i}(t) E_{i}, \\
\Delta_{i}(t) \Delta_{i}^{T}(t) & \leq I
\end{aligned}
$$

where $F_{i}$ and $E_{i}$ are known real constant matrices with appropriate dimensions, $\Delta_{i}(t)$ is an unknown time varying matrix function.

Assumption 2. The interconnected term $f_{i}(t, x, p)$ satisfies

$$
\left|f_{i}(t, x, p)\right| \leq k_{i}^{o}+\sum_{j=1}^{N} k_{i j}^{1}\left|x_{j}\right|+\sum_{j=1}^{N} k_{i j}^{2}\left|x_{i}\right|\left|x_{j}\right|,
$$

where $k_{i}^{o}, k_{i j}^{1}$, and $k_{i j}^{2}$ are unknown positive constants.

Now we show the general description of the quantizer $Q(\cdot)$. For the variable $s_{i} \in \mathbb{R}^{q_{i}}$, the quantizer operator $Q_{i}\left(s_{i}\right)$ is defined by a mathematical function $\operatorname{round}\left(s_{i}\right)$ that rounds the elements of $s_{i}$ towards the nearest integers; namely,

$$
\begin{aligned}
Q_{i}\left(s_{i}\right) & =\tau_{i d}(t) \cdot \operatorname{round}\left(\frac{s_{i}}{\tau_{i c}(t)}\right) \\
& :=\tau_{i d}(t) q_{i}\left(\frac{s_{i}}{\tau_{i c}(t)}\right)
\end{aligned}
$$

where $\tau_{i c}(t)$ and $\tau_{i d}(t)$ are the quantization sensitivity parameters at the coder and decoder sides of the $i$ th subsystem, respectively. During the operation process, the information of quantized measurement $q_{i}\left(s_{i} / \tau_{i c}(t)\right)$ is generated at the coder side of $i$ th subsystem; then it is sent to the decoder side over a communication channel. While at the decoder side of $i$ th subsystem, under the assumption that the channel is ideal, the value of quantized measurement $q_{i}\left(s_{i} / \tau_{i c}(t)\right)$ is received, and the quantization sensitivity parameter of the quantizer $\tau_{i d}(t)$ is adopted; then the decoder of $i$ th subsystem generates the signal $\tau_{i d}(t) q_{i}\left(s_{i} / \tau_{i c}(t)\right)$, that is, the quantization operator $Q_{i}\left(s_{i}\right)$.

Usually as done in $[1,2]$, it is assumed that the quantization parameters $\tau_{i c}(t)$ and $\tau_{i d}(t)$ are equal. In [17], the robustness of quantized control systems with respect to the mismatch of quantizer parameters is firstly presented. However, the adjustments of $\tau_{i c}(t)$ and $\tau_{i d}(t)$ are required to be synchronized at each instant. That is to say, $\tau_{i d}(t) / \tau_{i c}(t)=$ $r_{i}$, where $r_{i}$ is a time-invariant parameter. In actual control engineering, both of the requirements are obviously quite strict and hard to be implemented due to the hardware imperfections. For this, we establish the following timevarying ratio parameter model for the $i$ th subsystem:

$$
r_{i}(t):=\frac{\tau_{i d}(t)}{\tau_{i c}(t)}
$$

where $r_{i}(t) \in\left(r_{i \min }, r_{i \max }\right), r_{i \min }$, and $r_{i \max }$, satisfying $r_{i \max } \geq$ $r_{i \text { min }}$, are unknown positive scalars. 
Compared with our existing result in our previous result [20], the established model is more general since the lower and upper boundaries cannot be known.

Remark 3. Usually, besides quantization, signals transmitted over the channels also assume discretization on time, such as in $[4,14]$. To focus on the control design for the mismatch problem of quantizer sensitivity, only signal quantization is considered in this paper. Similar discusses were also made in $[13,15,19]$.

The main aim of this paper is to form decentralized adaptive integral sliding mode control strategy with $\mathscr{L}_{2}$ performance for the nonlinear large-scale system (1) subject to the quantization mismatch as described in (6).

Now considering $Q_{i}\left(u_{i}(t)\right)$ in (1) and the ratio in (6), we have

$$
\begin{aligned}
Q_{i}\left(u_{i}(t)\right) & =\tau_{i d}(t) q_{i}\left(\frac{u_{i}(t)}{\tau_{i c}(t)}\right) \\
& =\frac{\tau_{i d}(t)}{\tau_{i c}(t)} \tau_{i c}(t) q_{i}\left(\frac{u_{i}(t)}{\tau_{i c}(t)}\right) \\
& =r_{i}(t) \tau_{i c}(t) q_{i}\left(\frac{u_{i}(t)}{\tau_{i c}(t)}\right) \\
& =r_{i}(t)\left(u_{i}(t)+e_{\tau_{i c}}(t)\right),
\end{aligned}
$$

where $e_{\tau_{i c}}(t):=q_{\tau_{i c}(t)}\left(u_{i}(t)\right)-u_{i}(t)$ and $q_{\tau_{i c}(t)}\left(u_{i}(t)\right):=$ $\tau_{i c}(t) q_{i}\left(u_{i}(t) / \tau_{i c}(t)\right)$. And $e_{\tau_{i c}}(t)$ satisfies

$$
\left|e_{\tau_{i c}}(t)\right| \leq \nabla_{i} \tau_{i c}
$$

with $\nabla_{i}=\sqrt{m_{i}} / 2 ; m_{i}$ is the dimension of the vector $u_{i}$.

Definition 4. $\mathscr{L}_{2}$ Gain Performance. Given positive scalars $\gamma_{i}$, the nonlinear large-scale system in (1)-(2) with $u_{i}(t)=0$ is said to be robust stable with a bounded $\mathscr{L}_{2}$ gain performance; if it is robust stable for $\omega_{i}(t)=0$, and, under zero initial condition, for nonzero $\omega_{i}(t) \in \mathscr{L}_{2}[0, \infty)$, it holds that

$$
\sum_{i=1}^{N} \int_{0}^{\infty} z_{i}^{T}(t) z_{i}(t) d t<\sum_{i=1}^{N} \gamma_{i}^{2} \int_{0}^{\infty} \omega_{i}^{T}(t) \omega_{i}(t) d t
$$

Remark 5. Denote that

$$
\begin{aligned}
& z(t)=\left[z_{1}^{T}(t), z_{2}^{T}(t), \ldots, z_{N}^{T}(t)\right]^{T}, \\
& \omega(t)=\left[\omega_{1}^{T}(t), \omega_{2}^{T}(t), \ldots, \omega_{N}^{T}(t)\right]^{T},
\end{aligned}
$$

and letting $\gamma_{i}=\gamma, i=1,2, \ldots, N$, one can easily obtain that

$$
\int_{0}^{\infty} z^{T}(t) z(t) d t<\gamma^{2} \int_{0}^{\infty} \omega^{T}(t) \omega(t) d t .
$$

This is the usually considered $\mathscr{L}_{2}$ gain performance for simple linear system as in [26]. used.

For the considered problem, the following lemma will be
Lemma 6 (see [27]). Given a symmetric matrix $\Theta$ and matrices $X, Y$ with appropriate dimensions, then

$$
\Theta+X \Xi(t) Y+Y^{T} \Xi^{T}(t) X^{T}<0
$$

for all $\Xi(t)$ satisfying $\Xi^{T}(t) \Xi(t) \leq I$, if and only if there exists a scalar $\epsilon>0$ such that the following inequality holds:

$$
\Theta+\epsilon X X^{T}+\epsilon^{-1} Y^{T} Y<0 .
$$

\section{Main Results}

In this paper, the following integral-type sliding surface function is considered:

$$
\sigma_{i}(t)=G_{i} x_{i}(t)-\int_{0}^{t} G_{i}\left(A_{i}-B_{i} K_{i}\right) x_{i}\left(\theta_{i}\right) d \theta_{i},
$$

where $A_{i}$ and $B_{i}$ are system matrices defined in large-scale system (1). $G_{i} \in \mathbb{R}^{m_{i} \times n_{i}}$ and $K_{i} \in \mathbb{R}^{m_{i} \times n_{i}}$ are real matrices to be designed. In particular, the matrix $G_{i}$ is selected such that $G_{i} B_{i}$ is nonsingular. Without loss of generality, as done in $[28,29], G_{i}$ is designed to be $G_{i}=B_{i}^{+}$, the pseudoinverse of $B_{i}$ for the convenience of the proof.

By the theory of sliding mode control [30, 31], when the trajectory of large-scale system (1) is kept on the sliding dynamics, it has $\sigma_{i}(t)=0$ and $\dot{\sigma}_{i}(t)=0$. Thus, by taking $\dot{\sigma}_{i}(t)=0$, one can obtain the equivalent control for the $i$ th subsystem of large-scale system (1):

$$
\begin{aligned}
& Q_{\text {ieq }}\left(u_{i}(t)\right)=-\left[G_{i} B_{i}\right]^{-1}\left\{G_{i} \Delta A_{i}(t) x_{i}(t)\right. \\
& \left.\quad+G_{i} B_{i} f_{i}(t, x, p)+G_{i} B_{i} K_{i} x_{i}(t)+G_{i} D_{i} \omega_{i}(t)\right\} \\
& \quad=-\left(G_{i} B_{i}\right)^{-1} G_{i} \Delta A_{i}(t) x_{i}(t)-f_{i}(t, x, p)-K_{i} x_{i}(t) \\
& \quad-\left(G_{i} B_{i}\right)^{-1} G_{i} D_{i} \omega_{i}(t) .
\end{aligned}
$$

Substituting (15) into (1), one can get that

$$
\begin{aligned}
\dot{x}_{i}( & t)=\left(A_{i}+\Delta A_{i}(t)\right) x_{i}(t) \\
+ & B_{i}\left\{-\left(G_{i} B_{i}\right)^{-1} G_{i} \Delta A_{i}(t) x_{i}(t)-f_{i}(t, x, p)\right. \\
& \left.-K_{i} x_{i}(t)-\left(G_{i} B_{i}\right)^{-1} G_{i} D_{i} \omega_{i}(t)\right\}+B_{i} f_{i}(t, x, p) \\
& +D_{i} \omega_{i}(t)=\left[\left(A_{i}-B_{i} K_{i}\right)\right. \\
& \left.+\left(I-B_{i}\left(G_{i} B_{i}\right)^{-1} G_{i}\right) \Delta A_{i}(t)\right] x_{i}(t)+[I \\
& \left.-B_{i}\left(G_{i} B_{i}\right)^{-1} G_{i}\right] D_{i} \omega_{i}(t) .
\end{aligned}
$$

Denoting $\bar{G}_{i}=I-B_{i}\left(G_{i} B_{i}\right)^{-1} G_{i}, \bar{A}_{i}=A_{i}-B_{i} K_{i}, \Delta \bar{A}_{i}(t)=$ $\bar{G}_{i} \Delta A_{i}(t)$, and $\bar{D}_{i}=\bar{G}_{i} D_{i}$, one can see that (16) can be represented as

$$
\dot{x}_{i}(t)=\left[\bar{A}_{i}+\Delta \bar{A}_{i}(t)\right] x_{i}(t)+\bar{D}_{i} \omega_{i}(t),
$$

where $\Delta \bar{A}_{i}(t)=\bar{G}_{i} \Delta A_{i}(t)=\bar{G}_{i} F_{i} \Delta_{i}(t) E_{i}$.

We have the following theorem for the sliding mode dynamics in (17). 
Theorem 7. Given scalars $\gamma_{i}>0$, the sliding mode dynamics in (17) is quadratically stable with bounded $\mathscr{L}_{2}$ gain performance $\gamma_{i}$, if there exist a symmetric positive definite matrix $X_{i}, a$ general matrix $W_{i}$, and a positive scalar $\varepsilon_{i}$ such that the following linear matrix inequality holds:

$$
\left[\begin{array}{cccc}
\operatorname{He}\left\{A_{i} X_{i}-B_{i} W_{i}\right\}+\varepsilon_{i} \bar{G}_{i} F_{i} F_{i}^{T} \bar{G}_{i}^{T} & X_{i} C_{i}^{T} & X_{i} E_{i}^{T} & \bar{D}_{i}+X_{i} C_{i}^{T} H_{i} \\
* & -I & 0 & 0 \\
* & * & -\varepsilon_{i} I & 0 \\
* & * & * & H_{i}^{T} H_{i}-\gamma_{i}^{2} I
\end{array}\right]
$$

$<0$.

Moreover, if the LMI condition has a feasible solution, then the matrices $K_{i}$ in (14) can be derived by

$$
K_{i}=W_{i} X_{i}^{-1}
$$

Proof. Consider a Lyapunov function candidate as

$$
\bar{V}(t)=\sum_{i=1}^{N} \bar{V}_{i}(t)=\sum_{i=1}^{N} x_{i}^{T}(t) P_{i} x_{i}(t)
$$

where $P_{i}>0$. Along the solution of sliding mode dynamics (17), one can get

$$
\begin{aligned}
\dot{\bar{V}}(t) & =\sum_{i=1}^{N} \dot{\bar{V}}_{i}=\sum_{i=1}^{N} x_{i}^{T}(t) P_{i} \dot{x}_{i}(t)+\sum_{i=1}^{N} \dot{x}_{i}^{T}(t) P_{i} x_{i}(t) \\
= & \sum_{i=1}^{N} x_{i}^{T}(t) P_{i}\left\{\left[\bar{A}_{i}+\Delta \bar{A}_{i}(t)\right] x_{i}(t)+\bar{D}_{i} \omega_{i}(t)\right\} \\
& +\sum_{i=1}^{N}\left\{\left[\bar{A}_{i}+\Delta \bar{A}_{i}(t)\right] x_{i}(t)+\bar{D}_{i} \omega_{i}(t)\right\}^{T} P_{i} x_{i}(t) .
\end{aligned}
$$

Therefore,

$$
\begin{gathered}
\dot{\bar{V}}(t)+\sum_{i=1}^{N} z_{i}^{T}(t) z_{i}(t)-\sum_{i=1}^{N} \gamma_{i}^{2} \omega_{i}^{T}(t) \omega_{i}(t)=\sum_{i=1}^{N} x_{i}^{T}(t) \\
\cdot\left[\left(\bar{A}_{i}+\Delta \bar{A}_{i}(t)\right)^{T} P_{i}+P_{i}\left(\bar{A}_{i}+\Delta \bar{A}_{i}(t)\right)\right] x_{i}(t)
\end{gathered}
$$

$$
\begin{aligned}
& +\sum_{i=1}^{N} 2 x_{i}^{T}(t) P_{i} \bar{D}_{i} \omega_{i}(t)+\sum_{i=1}^{N}\left(C_{i} x_{i}(t)+H_{i} \omega_{i}(t)\right)^{T} \\
& \cdot\left(C_{i} x_{i}(t)+H_{i} \omega_{i}(t)\right)-\sum_{i=1}^{N} \gamma_{i}^{2} \omega_{i}^{T}(t) \omega_{i}(t) \\
& =\sum_{i=1}^{N} \xi_{i}^{T}(t) \Gamma_{i} \xi_{i}(t)
\end{aligned}
$$

where $\xi:=\left[\begin{array}{ll}x_{i}^{T}(t) & \omega_{i}^{T}(t)\end{array}\right]^{T}$ and

$$
\Gamma_{i}=\left[\begin{array}{cc}
\gamma_{11 i} & \gamma_{12 i} \\
* & \gamma_{22 i}
\end{array}\right]
$$

with $\gamma_{11 i}=\operatorname{He}\left[\left(\bar{A}_{i}+\Delta \bar{A}_{i}(t)\right)^{T} P_{i}\right]+C_{i}^{T} C_{i}, \gamma_{12 i}=P_{i} \bar{D}_{i}+C_{i}^{T} H_{i}$, and $\gamma_{22 i}=H_{i}^{T} H_{i}-\gamma_{i}^{2} I$.

Suppose that $\xi_{i}^{T}(t) \Gamma_{i} \xi_{i}(t)<0$, we have

$$
\dot{\bar{V}}(t)+\sum_{i=1}^{N} z_{i}^{T}(t) z_{i}(t)-\sum_{i=1}^{N} \gamma_{i}^{2} \omega_{i}^{T}(t) \omega_{i}(t)<0
$$

Integrating both sides of the inequality (24) with respect to time $t$ from 0 to $T$ yields

$$
\begin{aligned}
\bar{V}(T) & -\bar{V}(0)+\sum_{i=1}^{N} \int_{0}^{T} z_{i}^{T}(t) z_{i}(t) d t \\
& -\sum_{i=1}^{N} \gamma_{i}^{2} \int_{0}^{T} \omega_{i}^{T}(t) \omega_{i}(t) d t<0 .
\end{aligned}
$$

Under the zero initial conditions, we have $\bar{V}(0)=0$ and $\bar{V}(T)=\sum_{i=1}^{N} x_{i}^{T}(T) P_{i} x_{i}(T) \geq 0$; one can see that

$$
\sum_{i=1}^{N} \int_{0}^{T} z_{i}^{T}(t) z_{i}(t) d t<\sum_{i=1}^{N} \gamma_{i}^{2} \int_{0}^{T} \omega_{i}^{T}(t) \omega_{i}(t) d t
$$

Taking the limit as $T \rightarrow+\infty$ on both sides of (26) gives rise to (9).

Performing a congruence transformation to (23) by matrix $\operatorname{diag}\left\{P_{i}^{-1}, I\right\}$, we have

$$
\left[\begin{array}{cc}
\operatorname{He}\left\{\left[A_{i}-B_{i} K_{i}+G_{i} F_{i} \Delta_{i}(t) E_{i}\right] P_{i}^{-1}\right\}+P_{i}^{-1} C_{i}^{T} C_{i} P_{i}^{-1} & \bar{D}_{i}+P_{i}^{-1} C_{i}^{T} H_{i} \\
* & H_{i}^{T} H_{i}-\gamma_{i}^{2} I
\end{array}\right]<0 .
$$

Defining $X_{i}=P_{i}^{-1}$ and $W_{i}=K_{i} X_{i}$, we have

$$
\left[\begin{array}{cc}
\Theta_{11 i} & \bar{D}_{i}+X_{i} C_{i}^{T} H_{i} \\
* & H_{i}^{T} H_{i}-\gamma_{i}^{2} I
\end{array}\right]<0
$$

where $\Theta_{11 i}=\operatorname{He}\left\{A_{i} X_{i}-B_{i} W_{i}\right\}+G_{i} F_{i} \Delta_{i}(t) E_{i} X_{i}+$ $X_{i} E_{i}^{T} \Delta_{i}(t) F_{i}^{T} G_{i}^{T}+X_{i} C_{i}^{T} C_{i} X_{i}$. Furthermore, according to Lemma 6 , one can see that (28) is equivalent to

$$
\left[\begin{array}{cc}
\Xi_{11 i} & \bar{D}_{i}+X_{i} C_{i}^{T} H_{i} \\
* & H_{i}^{T} H_{i}-\gamma_{i}^{2} I
\end{array}\right]<0,
$$


where $\Xi_{11 i}=\operatorname{He}\left\{A_{i} X_{i}-B_{i} W_{i}\right\}+\varepsilon_{i} G_{i} F_{i} F_{i}^{T} G_{i}^{T}+$ $\left(1 / \varepsilon_{i}\right) X_{i} E_{i}^{T} E_{i} X_{i}+X_{i} C_{i}^{T} C_{i} X_{i}$. Applying Schur complement technique, we can further get (18).

This completes the proof.
In what follows, let us denote that $\sigma_{i}(t)=\left[\sigma_{i}^{1}(t)\right.$, $\left.\sigma_{i}^{2}(t), \ldots, \sigma_{i}^{m_{i}}(t)\right]^{T}$ and $u_{i}(t)=\left[u_{i}^{1}(t), u_{i}^{2}(t), \ldots, u_{i}^{m_{i}}(t)\right]^{T}$ for convenience. The following decentralized adaptive integral sliding mode control law is proposed:

$$
u_{i}^{j}(t)= \begin{cases}-\left[\beta_{i}\left(\widehat{l}_{i}+\widehat{\rho}_{i}\right)+\left[\left|G_{i} F_{i 1}\right|\left|E_{i}\right|+\left|K_{i}\right|\right]\right] \widehat{\gamma}_{i} \frac{\sigma_{i}^{j}(t)}{\left|\sigma_{i}(t)\right|}+u_{i}^{0}, & \text { if } \sigma_{i}^{j}(t)<0 \\ 0, & \text { if } \sigma_{i}^{j}(t)=0 \\ -\left[\beta_{i}\left(\widehat{l}_{i}+\hat{\rho}_{i}\right)+\left[\left|G_{i} F_{i 1}\right|\left|E_{i}\right|+\left|K_{i}\right|\right]\right] \widehat{\gamma}_{i} \frac{\sigma_{i}^{j}(t)}{\left|\sigma_{i}(t)\right|}-u_{i}^{0}, & \text { if } \sigma_{i}^{j}(t)>0,\end{cases}
$$

where $u_{i}^{0}=\nabla_{i} \tau_{i c}(t), \beta_{i}>1 . i=1,2, \ldots, N ; j=1,2, \ldots, m_{i}$. The parameters $\widehat{l}_{i}, \hat{\rho}_{i}>0$, and $\widehat{\gamma}_{i}>0$ are the estimations of $l_{i}, \rho_{i}$, and $r_{i}^{-1}$, respectively. And they are derived from the designed adaptive laws:

$$
\begin{aligned}
& \dot{\hat{l}}_{i}(t)=\frac{1}{\eta_{i 1}}\left|\sigma_{i}(t)\right|, \\
& \dot{\hat{\rho}}_{i}(t)=\frac{1}{\eta_{i 2}}\left|\sigma_{i}(t)\right|, \\
& \dot{\hat{\gamma}}_{i}=\frac{1}{\eta_{i 3}}\left(\hat{l}_{i}+\widehat{\rho}_{i}+\left[\left|G_{i} F_{i 1}\right|\left|E_{i}\right|+\left|K_{i}\right|\right]\left|x_{i}\right|\right) \hat{\gamma}_{i}^{3}\left|\sigma_{i}(t)\right|
\end{aligned}
$$

with the initial values $\widehat{l}_{i}(0)=\widehat{l}_{i 0}>0, \widehat{\gamma}_{i}(0)=\widehat{\gamma}_{i 0}>0$, and $\hat{\rho}_{i}(0)=\hat{\rho}_{i 0}>0$, and the parameters $\eta_{i 1}, \eta_{i 2}$, and $\eta_{i 3}$ are selected positive scalars; $i=1,2, \ldots, N . l_{i}$ and $\rho_{i}$ will be given later.

Remark 8. The controller form as (31)-(33) can be often seen in existing theory results, for instance, [23, 32]. However, it is hard to expect $|\sigma(t)|$ to be exactly zero in practice engineering; thus how to solve the gain increase problem in theory completely is a very interesting research topic. In addition, one can see from the simulation part later, by choosing sufficiently large parameters $\eta_{i 1}, \eta_{i 2}$, and $\eta_{i 3}$, the gains cannot increase indefinitely in a finite time. So they can be used in some engineering applications.

Remark 9. The solution for systems with discontinuous righthand side as (30) can be interpreted in the sense of [33-35].

To clarify the proof of the main result, a lemma is first summarized in the following.

Lemma 10. For the quantized control input $Q_{i}\left(u_{i}\right)$ satisfying the mismatched relation shown in (6), the control law in (30) guarantees the establishment of the inequality:

$$
\begin{aligned}
& \sigma_{i}^{T}(t) Q_{i}\left(u_{i}\right) \leq-r_{i}(t) \\
& \quad \cdot\left\{\beta_{i}\left(\widehat{l}_{i}+\widehat{\rho}_{i}\right)+\left[\left|G_{i} F_{i 1}\right|\left|E_{i}\right|+\left|K_{i}\right|\right]\left|x_{i}\right|\right\} \widehat{\gamma}_{i}\left|\sigma_{i}(t)\right| .
\end{aligned}
$$

Proof. It is obvious that the above inequality (34) holds when $\sigma_{i}(t)=0$. And when $\sigma_{i}^{j}(t)<0$, we have $u_{i}^{j}=-\left[\beta_{i}\left(\widehat{l}_{i}+\right.\right.$ $\left.\left.\hat{\rho}_{i}\right)+\left[\left|G_{i} F_{i}\right|\left|E_{i}\right|+\left|K_{i}\right|\right]\right] \widehat{\gamma}_{i}\left(\sigma_{i}^{j}(t) /\left|\sigma_{i}(t)\right|\right)+u_{i}^{0}$. Combining with the mismatched relation in (6), the definition of quantization error, one can see that

$$
\begin{aligned}
& \left(u_{i}^{j}-u_{i}^{0}\right) Q_{i}\left(u_{i}^{j}\right)=-\left[\beta_{i}\left(\widehat{l}_{i}+\widehat{\rho}_{i}\right)\right. \\
& \left.+\left[\left|G_{i} F_{i}\right|\left|E_{i}\right|+\left|K_{i}\right|\right]\right] \widehat{\gamma}_{i} \frac{\sigma_{i}^{j}(t)}{\left|\sigma_{i}(t)\right|} Q_{i}\left(u_{i}^{j}\right) \\
& =-\left[\beta_{i}\left(\widehat{l}_{i}+\widehat{\rho}_{i}\right)+\left[\left|G_{i} F_{i}\right|\left|E_{i}\right|+\left|K_{i}\right|\right]\right] \widehat{\gamma}_{i} \frac{\sigma_{i}^{j}(t)}{\left|\sigma_{i}(t)\right|} \\
& \cdot r_{i}(t)\left(u_{i}^{(j)}+e_{\tau_{i c}}^{j}(t)\right)=-\left[\beta_{i}\left(\widehat{l}_{i}+\widehat{\rho}_{i}\right)\right. \\
& \left.+\left[\left|G_{i} F_{i}\right|\left|E_{i}\right|+\left|K_{i}\right|\right]\right] \widehat{\gamma}_{i} \frac{\sigma_{i}^{j}(t)}{\left|\sigma_{i}(t)\right|} r_{i}(t) \\
& \cdot\left[-\left[\beta_{i}\left(\widehat{l}_{i}+\widehat{\rho}_{i}\right)+\left[\left|G_{i} F_{i}\right|\left|E_{i}\right|+\left|K_{i}\right|\right]\right] \widehat{\gamma}_{i} \frac{\sigma_{i}^{j}(t)}{\left|\sigma_{i}(t)\right|}\right. \\
& \left.+\nabla_{i} \tau_{i c}(t)+e_{\tau_{i c}}^{j}(t)\right] \\
& =\left\{-\left[\beta_{i}\left(\widehat{l}_{i}+\widehat{\rho}_{i}\right)+\left[\left|G_{i} F_{i}\right|\left|E_{i}\right|+\left|K_{i}\right|\right]\right] \widehat{\gamma}_{i}\right. \\
& \left.\cdot \frac{\sigma_{i}^{j}(t)}{\left|\sigma_{i}(t)\right|}\right\}^{2} r_{i}(t)-\left[\beta_{i}\left(\widehat{l}_{i}+\widehat{\rho}_{i}\right)\right. \\
& \left.+\left[\left|G_{i} F_{i}\right|\left|E_{i}\right|+\left|K_{i}\right|\right]\right] \widehat{\gamma}_{i} \frac{\sigma_{i}^{j}(t)}{\left|\sigma_{i}(t)\right|} r_{i}(t)\left[\nabla_{i} \tau_{i c}(t)\right. \\
& \left.+e_{\tau_{i c}}^{j}(t)\right] .
\end{aligned}
$$

By the design of the parameters, $\nabla_{i} \tau_{i c} \geq\left|e_{\tau_{i c}^{j}}^{j}(t)\right|$ and $\sigma_{i j}(t)<0$, one can see that

$$
\begin{aligned}
- & {\left[\beta_{i}\left(\widehat{l}_{i}+\widehat{\rho}_{i}\right)+\left[\left|G_{i} F_{i}\right|\left|E_{i}\right|+\left|K_{i}\right|\right]\right] \widehat{\gamma}_{i} \frac{\sigma_{i}^{j}(t)}{\left|\sigma_{i}(t)\right|} r_{i}(t) } \\
\cdot & {\left[\nabla_{i} \tau_{i c}(t)+e_{\tau_{i c}}^{j}(t)\right] \geq 0 . }
\end{aligned}
$$


Combining (35) with (36), we have

$$
\begin{aligned}
& \left(u_{i}^{j}-u_{i}^{0}\right) Q_{i}\left(u_{i}^{j}\right)=-\left[\beta_{i}\left(\widehat{l}_{i}+\widehat{\rho}_{i}\right)\right. \\
& \left.+\left[\left|G_{i} F_{i 1}\right|\left|E_{i}\right|+\left|K_{i}\right|\right]\right] \widehat{\gamma}_{i} \frac{\sigma_{i}^{j}(t)}{\left|\sigma_{i}(t)\right|} Q_{i}\left(u_{i}^{j}\right) \geq r_{i}(t) \\
& \quad \cdot\left\{-\left[\beta_{i}\left(\widehat{l}_{i}+\widehat{\rho}_{i}\right)+\left[\left|G_{i} F_{i 1}\right|\left|E_{i}\right|+\left|K_{i}\right|\right]\right] \widehat{\gamma}_{i} \frac{\sigma_{i}^{j}(t)}{\left|\sigma_{i}(t)\right|}\right\}^{2} .
\end{aligned}
$$

Since $-\left[\beta_{i}\left(\widehat{l}_{i}+\widehat{\rho}_{i}\right)+\left[\left|G_{i} F_{i}\right|\left|E_{i}\right|+\left|K_{i}\right|\right]\right] \widehat{\gamma}_{i}<0$, it is not difficult to get that

$$
\begin{aligned}
\sigma_{i}^{j}(t) Q_{i}\left(u_{i}^{j}\right) \leq & -r_{i}(t) \\
& \cdot\left[\beta_{i}\left(\widehat{l}_{i}+\widehat{\rho}_{i}\right)+\left[\left|G_{i} F_{i}\right|\left|E_{i}\right|+\left|K_{i}\right|\right]\right] \widehat{\gamma}_{i} \\
& \cdot \frac{\left(\sigma_{i}^{j}(t)\right)^{2}}{\left|\sigma_{i}(t)\right|} .
\end{aligned}
$$

Similarly, when $\sigma_{i}^{j}(t)>0$, we have $u_{i}^{j}=-\left[\beta_{i}\left(\widehat{l}_{i}+\widehat{\rho}_{i}\right)+\right.$ $\left.\left[\left|G_{i} F_{i}\right|\left|E_{i}\right|+\left|K_{i}\right|\right]\right] \widehat{\gamma}_{i}\left(\sigma_{i}^{j}(t) /\left|\sigma_{i}(t)\right|\right)-u_{i}^{0}$. Combining with the mismatched relation in (6), the definition of quantization error again, one can see that

$$
\begin{aligned}
& \left(u_{i}^{j}+u_{i}^{0}\right) Q_{i}\left(u_{i}^{j}\right)=-\left[\beta_{i}\left(\widehat{l}_{i}+\widehat{\rho}_{i}\right)\right. \\
& \left.+\left[\left|G_{i} F_{i}\right|\left|E_{i}\right|+\left|K_{i}\right|\right]\right] \widehat{\gamma}_{i} \frac{\sigma_{i}^{j}(t)}{\left|\sigma_{i}(t)\right|} Q_{i}\left(u_{i}^{j}\right) \\
& =-\left[\beta_{i}\left(\widehat{l}_{i}+\widehat{\rho}_{i}\right)+\left[\left|G_{i} F_{i}\right|\left|E_{i}\right|+\left|K_{i}\right|\right]\right] \widehat{\gamma}_{i} \frac{\sigma_{i}^{j}(t)}{\left|\sigma_{i}(t)\right|} \\
& \cdot r_{i}(t)\left(u_{i}^{j}(t)+e_{\tau_{i c}}^{j}(t)\right)=-\left[\beta_{i}\left(\widehat{l}_{i}+\widehat{\rho}_{i}\right)\right. \\
& \left.+\left[\left|G_{i} F_{i}\right|\left|E_{i}\right|+\left|K_{i}\right|\right]\right] \widehat{\gamma}_{i} \frac{\sigma_{i}^{j}(t)}{\left|\sigma_{i}(t)\right|} r_{i}(t) \\
& \cdot\left\{-\left[\beta_{i}\left(\widehat{l}_{i}+\widehat{\rho}_{i}\right)+\left[\left|G_{i} F_{i}\right|\left|E_{i}\right|+\left|K_{i}\right|\right]\right] \widehat{\gamma}_{i} \frac{\sigma_{i}^{j}(t)}{\left|\sigma_{i}(t)\right|}\right. \\
& \left.-\nabla_{i} \tau_{i c}(t)+e_{\tau_{i c}}^{j}(t)\right\} \\
& =\left\{-\left[\beta_{i}\left(\widehat{l}_{i}+\widehat{\rho}_{i}\right)+\left[\left|G_{i} F_{i}\right|\left|E_{i}\right|+\left|K_{i}\right|\right]\right] \widehat{\gamma}_{i}\right. \\
& \left.\frac{\sigma_{i}^{j}(t)}{\left|\sigma_{i}(t)\right|}\right\}^{2} r_{i}(t)+\left\{\beta_{i}\left(\widehat{l}_{i}+\widehat{\rho}_{i}\right)\right. \\
& \left.+\left[\left|G_{i} F_{i}\right|\left|E_{i}\right|+\left|K_{i}\right|\right]\right\} \widehat{\gamma}_{i} \frac{\sigma_{i}^{j}(t)}{\left|\sigma_{i}(t)\right|} r_{i}(t)\left[\nabla_{i} \tau_{i c}(t)\right. \\
& \left.-e_{\tau_{i c}}^{j}(t)\right] .
\end{aligned}
$$

According to the design of the parameters, $\nabla_{i} \tau_{i c} \geq\left|e_{\tau_{i c}}^{j}(t)\right|$ and $\sigma_{i}^{j}(t)>0$, one can see that

$$
\begin{aligned}
& {\left[\beta_{i}\left(\widehat{l}_{i}+\widehat{\rho}_{i}\right)+\left[\left|G_{i} F_{i}\right|\left|E_{i}\right|+\left|K_{i}\right|\right]\right] \widehat{\gamma}_{i} \frac{\sigma_{i}^{j}(t)}{\left|\sigma_{i}(t)\right|} r_{i}(t)} \\
& \cdot\left[\nabla_{i} \tau_{i c}(t)-e_{\tau_{i c}}^{j}(t)\right] \geq 0 .
\end{aligned}
$$

Combining (39) with (40), we have

$$
\begin{aligned}
& \left(u_{i}^{j}+u_{i}^{0}\right) Q_{i}\left(u_{i}^{j}\right) \\
& =-\left[\beta_{i}\left(\widehat{l}_{i}+\widehat{\rho}_{i}\right)+\left[\left|G_{i} F_{i}\right|\left|E_{i}\right|+\left|K_{i}\right|\right]\right] \widehat{\gamma}_{i} \frac{\sigma_{i}^{j}(t)}{\left|\sigma_{i}(t)\right|} \\
& \cdot Q_{i}\left(u_{i}^{j}\right) \\
& \geq\left[-\left[\beta_{i}\left(\widehat{l}_{i}+\widehat{\rho}_{i}\right)+\left[\left|G_{i} F_{i}\right|\left|E_{i}\right|+\left|K_{i}\right|\right]\right] \widehat{\gamma}_{i} \frac{\sigma_{i}^{j}(t)}{\left|\sigma_{i}(t)\right|}\right]^{2} \\
& \cdot r_{i}(t) .
\end{aligned}
$$

Furthermore, we have

$$
\begin{aligned}
\sigma_{i}^{j}(t) Q_{i}\left(u_{i}^{j}\right) \leq & -r_{i}(t) \\
& \cdot\left[\beta_{i}\left(\widehat{l}_{i}+\widehat{\rho}_{i}\right)+\left[\left|G_{i} F_{i}\right|\left|E_{i}\right|+\left|K_{i}\right|\right]\right] \widehat{\gamma}_{i} \\
& \cdot \frac{\left(\sigma_{i}^{j}(t)\right)^{2}}{\left|\sigma_{i}(t)\right|} .
\end{aligned}
$$

Therefore, the following inequality holds:

$$
\begin{aligned}
& \sigma_{i}^{T}(t) Q_{i}\left(u_{i}\right)=\sum_{j=1}^{m} \sigma_{i j}(t) Q_{i}\left(u_{i}^{j}\right) \leq-\sum_{j=1}^{m} r_{i}(t) \\
& \cdot\left[\beta_{i}\left(\widehat{l}_{i}+\widehat{\rho}_{i}\right)+\left[\left|G_{i} F_{i}\right|\left|E_{i}\right|+\left|K_{i}\right|\right]\right] \widehat{\gamma}_{i} \frac{\left(\sigma_{i}^{j}(t)\right)^{2}}{\left|\sigma_{i}(t)\right|} \\
& \leq-\left[\beta_{i}\left(\widehat{l}_{i}+\widehat{\rho}_{i}\right)+\left[\left|G_{i} F_{i}\right|\left|E_{i}\right|+\left|K_{i}\right|\right]\right] \\
& \cdot \widehat{\gamma}_{i} \sum_{j=1}^{m}\left[\frac{\left(\sigma_{i}^{j}(t)\right)^{2}}{\left|\sigma_{i}(t)\right|}\right]=-r_{i}(t)\left[\beta_{i}\left(\widehat{l}_{i}+\widehat{\rho}_{i}\right)\right. \\
& \left.+\left[\left|G_{i} F_{i}\right|\left|E_{i}\right|+\left|K_{i}\right|\right]\right] \widehat{\gamma}_{i}\left|\sigma_{i}(t)\right| .
\end{aligned}
$$

The proof has been achieved completely.

The main result of this paper is summarized as follows.

Theorem 11. Consider the nonlinear large-scale system (1) subject to Assumptions 1-2 with the mismatch between the quantization sensitivity parameters; if the control law is designed as shown in (30) with adaptive laws in (31)-(33), then the trajectories of system (1) can be driven onto the sliding surfaces asymptotically. 
Proof. Let us first consider $V_{i}(t)=(1 / 2) \sigma_{i}^{T}(t) \sigma_{i}(t)$, then the time derivative along the system trajectories is

$$
\begin{aligned}
& \dot{V}_{i}(t)=\sigma_{i}^{T}(t)\left\{G_{i} \Delta A_{i}(t) x_{i}+G_{i} B_{i} Q_{i}\left(u_{i}\right)\right. \\
& \left.+G_{i} B_{i} f_{i}(t, x, p)+G_{i} B_{i} K_{i} x_{i}+G_{i} D_{i} \omega_{i}(t)\right\} .
\end{aligned}
$$

According to the design of the switching vector $G_{i}=B_{i}^{+}$, one can see that the above equation can be rewritten to be

$$
\begin{aligned}
& \dot{V}_{i}(t)=\sigma_{i}^{T}(t) Q_{i}\left(u_{i}(t)\right)+\sigma_{i}^{T}(t) \\
& \quad \cdot\left\{G_{i} \Delta A_{i}(t) x_{i}+f_{i}(t, x, p)+K_{i} x_{i}+G_{i} D_{i} \omega_{i}(t)\right\} .
\end{aligned}
$$

By Assumptions 1-2, we have

$$
\begin{aligned}
\dot{V}_{i}(t) \leq & \sigma_{i}^{T}(t) Q_{i}\left(u_{i}(t)\right)+\left|\sigma_{i}(t)\right|\left|G_{i} F_{i}\right|\left|E_{i}\right|\left|x_{i}\right| \\
& +\left|\sigma_{i}(t)\right|\left|K_{i}\right|\left|x_{i}\right|+\left|\sigma_{i}(t)\right|\left|G_{i} D_{i}\right|\left|\omega_{i}(t)\right| \\
& +\left|\sigma_{i}(t)\right|\left[k_{i}^{0}+\sum_{j=1}^{N} k_{i j}^{1}\left|x_{j}\right|+\sum_{j=1}^{N} k_{i j}^{2}\left|x_{i}\right|\left|x_{j}\right|\right] .
\end{aligned}
$$

Applying the basic inequality $a b \leq(1 / 2)\left(a^{2}+b^{2}\right), a \in \mathbb{R}$, $b \in \mathbb{R}$, one can see that

$$
\begin{aligned}
\dot{V}_{i}(t) & \\
\leq & \sigma_{i}^{T}(t) Q_{i}\left(u_{i}(t)\right)+\left|\sigma_{i}(t)\right|\left|G_{i} F_{i}\right|\left|E_{i}\right|\left|x_{i}\right| \\
& +\left|\sigma_{i}(t)\right|\left|K_{i}\right|\left|x_{i}\right|+\left|\sigma_{i}(t)\right|\left|G_{i} D_{i}\right|\left|\omega_{i}(t)\right| \\
+ & \left|\sigma_{i}(t)\right|\left[k_{i}^{0}+\sum_{j=1}^{N} k_{i j}^{1}\left|x_{j}\right|+\frac{1}{2} \sum_{j=1}^{N} k_{i j}^{2}\left(x_{i}^{2}+x_{j}^{2}\right)\right] \\
= & \sigma_{i}^{T}(t) Q_{i}\left(u_{i}(t)\right)+\left|\sigma_{i}(t)\right|\left[\left|G_{i} F_{i}\right|\left|E_{i}\right|+\left|K_{i}\right|\right]\left|x_{i}\right| \\
+ & {\left[\sigma_{i}(t) \mid\left[\left|G_{i} D_{i}\right|\left|\omega_{i}(t)\right|+k_{i}^{0}+\frac{1}{2} \sum_{j=1}^{N} k_{i j}^{2} x_{i}^{2}\right]\right.} \\
+ & {\left[\sigma_{i}(t) \mid\left[\sum_{j=1}^{N} k_{i j}^{1}\left|x_{j}\right|+\frac{1}{2} \sum_{j=1}^{N} k_{i j}^{2} x_{j}^{2}\right] .\right.}
\end{aligned}
$$

Since $k_{i}^{0}, k_{i j}^{1}$, and $k_{i j}^{2}$ are unknown parameters, for convenience, similar to what is done in [23], we denote that unknown parameters $l_{i}$ and $\rho_{i}$ satisfy

$$
\begin{aligned}
& l_{i} \geq\left|G_{i} D_{i}\right|\left|\omega_{i}(t)\right|+k_{i}^{0}+\frac{1}{2} \sum_{j=1}^{N} k_{i j}^{2} x_{i}^{2}, \\
& \rho_{i} \geq \sum_{j=1}^{N} k_{i j}^{1}\left|x_{j}\right|+\frac{1}{2} \sum_{j=1}^{N} k_{i j}^{2} x_{j}^{2} ;
\end{aligned}
$$

then (47) can be rewritten as

$$
\begin{aligned}
\dot{V}_{i}(t) & \\
\leq & \sigma_{i}^{T}(t) Q_{i}\left(u_{i}(t)\right)+\left|\sigma_{i}(t)\right|\left|G_{i} F_{i}\right|\left|E_{i}\right|\left|x_{i}\right| \\
& +\left|\sigma_{i}(t)\right|\left|K_{i}\right|\left|x_{i}\right|+\left|\sigma_{i}(t)\right|\left|G_{i} D_{i}\right|\left|\omega_{i}(t)\right| \\
& +\left|\sigma_{i}(t)\right|\left[k_{i}^{0}+\sum_{j=1}^{N} k_{i j}^{1}\left|x_{j}\right|+\frac{1}{2} \sum_{j=1}^{N} k_{i j}^{2}\left(x_{i}^{2}+x_{j}^{2}\right)\right] \\
= & \sigma_{i}^{T}(t) Q_{i}\left(u_{i}(t)\right)+\left|\sigma_{i}(t)\right|\left[\left[G_{i} F_{i}\right]\left|E_{i}\right|+\left|K_{i}\right|\right]\left|x_{i}\right| \\
& +\left|\sigma_{i}(t)\right| l_{i}+\left|\sigma_{i}(t)\right| \rho_{i} .
\end{aligned}
$$

Take Lyapunov function candidate as

$$
V(t)=\sum_{i=1}^{N} V_{i}(t)+\frac{1}{2} \sum_{i=1}^{N} \eta_{i 1} \widetilde{l}_{i}^{2}+\frac{1}{2} \sum_{i=1}^{N} \eta_{i 2} \widetilde{\rho}_{i}^{2}+\frac{1}{2} \sum_{i=1}^{N} \eta_{i 3} \widetilde{\gamma}_{i}^{2},
$$

where $\widetilde{l}_{i}=l_{i}-\widehat{l}_{i}, \tilde{\rho}_{i}=\rho_{i}-\widehat{\rho}_{i}$, and $\tilde{\gamma}_{i}=\widehat{\gamma}_{i}^{-1}-r_{i}$. Thus we have

$$
\begin{aligned}
\dot{V}(t) & =\sum_{i=1}^{N} \dot{V}_{i}(t)+\sum_{i=1}^{N} \eta_{i 1} \tilde{\widetilde{l}}_{i} \dot{\bar{l}}_{i}+\sum_{i=1}^{N} \eta_{i 2} \widetilde{\rho}_{i} \dot{\tilde{\rho}}_{i}+\sum_{i=1}^{N} \eta_{i 3} \widetilde{\gamma}_{i} \dot{\tilde{\gamma}}_{i} \\
& =\sum_{i=1}^{N}\left\{\sigma_{i}^{T}(t) Q_{i}\left(u_{i}(t)\right)\right. \\
& +\left|\sigma_{i}(t)\right|\left[\left|G_{i} F_{i}\right|\left|E_{i}\right|+\left|K_{i}\right|\right]\left|x_{i}\right|+\left|\sigma_{i}(t)\right| l_{i} \\
& \left.+\left|\sigma_{i}(t)\right| \rho_{i}\right\}+\sum_{i=1}^{N} \eta_{i 1} \tilde{l}_{i} \dot{\vec{l}}_{i}+\sum_{i=1}^{N} \eta_{i 2} \tilde{\rho}_{i} \dot{\tilde{\rho}}_{i}+\sum_{i=1}^{N} \eta_{i 3} \widetilde{\gamma}_{i} \dot{\tilde{\gamma}}_{i}
\end{aligned}
$$

According to the design of the adaptive laws (31)-(33) and combining with $\widetilde{l}_{i}=l_{i}-\widehat{l}_{i}, \tilde{\rho}_{i}=\rho_{i}-\widehat{\rho}_{i}$, and $\widetilde{\gamma}_{i}=\widehat{\gamma}_{i}^{-1}-r_{i}$, we have

$$
\begin{aligned}
\dot{V}( & t)=\sum_{i=1}^{N} \dot{V}_{i}(t)+\sum_{i=1}^{N} \eta_{i 1} \tilde{l}_{i} \dot{\vec{l}}_{i}+\sum_{i=1}^{N} \eta_{i 2} \widetilde{\rho}_{i} \dot{\tilde{\rho}}_{i}+\sum_{i=1}^{N} \eta_{i 3} \widetilde{\gamma}_{i} \dot{\tilde{\gamma}}_{i} \\
= & \sum_{i=1}^{N}\left\{\sigma_{i}^{T}(t) Q_{i}\left(u_{i}(t)\right)\right. \\
+ & \left|\sigma_{i}(t)\right|\left[\left|G_{i} F_{i}\right|\left|E_{i}\right|+\left|K_{i}\right|\right]\left|x_{i}\right|+\left|\sigma_{i}(t)\right| \widehat{l}_{i} \\
& \left.+\left|\sigma_{i}(t)\right| \hat{\rho}_{i}\right\}+\sum_{i=1}^{N} \eta_{i 3} \widetilde{\gamma}_{i}\left(-\frac{1}{\gamma_{i}^{2}}\right) \dot{\hat{\gamma}}_{i} \\
& =\sum_{i=1}^{N}\left\{\sigma_{i}^{T}(t) Q_{i}\left(u_{i}(t)\right)\right. \\
& +\left|\sigma_{i}(t)\right|\left[\left|G_{i} F_{i}\right|\left|E_{i}\right|+\left|K_{i}\right|\right]\left|x_{i}\right|+\left|\sigma_{i}(t)\right| \widehat{l}_{i} \\
& \left.+\left|\sigma_{i}(t)\right| \hat{\rho}_{i}\right\}-\sum_{i=1}^{N} \tilde{\gamma}_{i} \widehat{\gamma}_{i}\left(\widehat{l}_{i}+\widehat{\rho}_{i}\right. \\
& \left.+\left[\left|G_{i} F_{i}\right|\left|E_{i}\right|+\left|K_{i}\right|\right]\left|x_{i}\right|\right)\left|\sigma_{i}(t)\right| .
\end{aligned}
$$


According to Lemma 10, one can see that

$\dot{V}(t)$

$$
\begin{aligned}
& \leq \sum_{i=1}^{N}\left\{-r_{i} \widehat{\gamma}_{i}\left(\beta_{i}\left(\widehat{l}_{i}+\widehat{\rho}_{i}\right)+\left[\left|G_{i} F_{i}\right|\left|E_{i}\right|+\left|K_{i}\right|\right]\left|x_{i}\right|\right)\right. \\
& \cdot\left|\sigma_{i}(t)\right|+\left|\sigma_{i}(t)\right|\left[\left|G_{i} F_{i 1}\right|\left|E_{i}\right|+\left|K_{i}\right|\right]\left|x_{i}\right|+\left|\sigma_{i}(t)\right| \widehat{l}_{i} \\
& \left.+\left|\sigma_{i}(t)\right| \widehat{\rho}_{i}\right\}-\sum_{i=1}^{N} \widetilde{\gamma}_{i} \widehat{\gamma}_{i}\left(\widehat{l}_{i}+\widehat{\rho}_{i}+\left[\left|G_{i} F_{i}\right|\left|E_{i}\right|+\left|K_{i}\right|\right]\right. \\
& \left.\cdot\left|x_{i}\right|\right)\left|\sigma_{i}(t)\right| .
\end{aligned}
$$

Furthermore, noticing that $\tilde{\gamma}_{i}=\widehat{\gamma}_{i}^{-1}-\gamma_{i}, \beta_{i}>1$, and $\widehat{l}_{i}>0$, $\hat{\rho}_{i}>0$, one can easily obtain that

$$
\begin{aligned}
& \dot{V}(t) \leq \sum_{i=1}^{N}\left\{-\left(r_{i}+\tilde{\gamma}_{i}\right)\right. \\
& \cdot \widehat{\gamma}_{i}\left(\beta_{i}\left(\widehat{l}_{i}+\widehat{\rho}_{i}\right)+\left[\left|G_{i} F_{i}\right|\left|E_{i}\right|+\left|K_{i}\right|\right]\left|x_{i}\right|\right)\left|\sigma_{i}(t)\right| \\
& +\left|\sigma_{i}(t)\right|\left[\left|G_{i} F_{i}\right|\left|E_{i}\right|+\left|K_{i}\right|\right]\left|x_{i}\right|+\left|\sigma_{i}(t)\right| \widehat{l}_{i}+\left|\sigma_{i}(t)\right| \\
& \left.\cdot \widehat{\rho}_{i}\right\}=\sum_{i=1}^{N}\left\{-\left(\beta_{i}\left(\widehat{l}_{i}+\widehat{\rho}_{i}\right)+\left[\left|G_{i} F_{i}\right|\left|E_{i}\right|+\left|K_{i}\right|\right]\left|x_{i}\right|\right)\right. \\
& \cdot\left|\sigma_{i}(t)\right|+\left|\sigma_{i}(t)\right|\left[\left|G_{i} F_{i}\right|\left|E_{i}\right|+\left|K_{i}\right|\right]\left|x_{i}\right|+\left|\sigma_{i}(t)\right| \widehat{l}_{i} \\
& \left.+\left|\sigma_{i}(t)\right| \hat{\rho}_{i}\right\}=-\sum_{i=1}^{N}\left(\beta_{i}-1\right)\left(\widehat{l}_{i}+\widehat{\rho}_{i}\right)\left|\sigma_{i}(t)\right|<0 .
\end{aligned}
$$

Integrating both sides of the above equation from 0 to $t$ gives rise to

$$
V(t)-V(0) \leq \sum_{i=1}^{N} \int_{0}^{t}\left(1-\beta_{i}\right)\left(\widehat{l}_{i}+\widehat{\rho}_{i}\right)\left|\sigma_{i}(s)\right| d s .
$$

One can easily obtain that

$$
\sum_{i=1}^{N} \int_{0}^{t}\left(\beta_{i}-1\right)\left(\widehat{l}_{i}+\widehat{\rho}_{i}\right)\left|\sigma_{i}(s)\right| d s \leq V(0) .
$$

Taking the limit as $t \rightarrow+\infty$ on both sides of (56) yields

$$
\sum_{i=1}^{N} \int_{0}^{\infty}\left(\beta_{i}-1\right)\left(\widehat{l}_{i}+\widehat{\rho}_{i}\right)\left|\sigma_{i}(s)\right| d s \leq V(0)<+\infty
$$

According to Barbalat's lemma, one can see that

$$
\lim _{t \rightarrow+\infty} \sum_{i=1}^{N}\left(\beta_{i}-1\right)\left(\widehat{l}_{i}+\widehat{\rho}_{i}\right)\left|\sigma_{i}(s)\right|=0 .
$$

Since $\beta_{i}>1, \widehat{l}_{i}>0$, and $\widehat{\rho}_{i}>0$ are positive increasing functions in terms of the adaptive control laws in (31) and (32), thus we have $\sigma_{i}(t) \rightarrow 0$ as $t \rightarrow+\infty$.

Hence the proof is obtained completely.

\section{Illustrative Example}

Consider the nonlinear uncertain large-scale system (1) with the following data:

$$
\begin{aligned}
& A_{1}=\left[\begin{array}{cc}
-2 & 3 \\
2 & 1
\end{array}\right] \text {, } \\
& A_{2}=\left[\begin{array}{cc}
-1 & 1 \\
0 & -2
\end{array}\right] \text {, } \\
& B_{1}=\left[\begin{array}{l}
1 \\
2
\end{array}\right] \text {, } \\
& B_{2}=\left[\begin{array}{l}
-1 \\
0.5
\end{array}\right] \text {, } \\
& F_{1}=\left[\begin{array}{l}
1 \\
1
\end{array}\right] \text {, } \\
& F_{2}=\left[\begin{array}{l}
0 \\
1
\end{array}\right] \text {, } \\
& E_{1}=\left[\begin{array}{ll}
0 & 1
\end{array}\right] \text {, } \\
& E_{2}=\left[\begin{array}{ll}
1 & 1
\end{array}\right] \text {, } \\
& D_{1}=\left[\begin{array}{l}
1 \\
1
\end{array}\right] \text {, } \\
& D_{2}=\left[\begin{array}{c}
-2 \\
1
\end{array}\right] \text {, } \\
& C_{1}=\left[\begin{array}{ll}
1 & 2
\end{array}\right] \text {, } \\
& C_{2}=\left[\begin{array}{ll}
2 & 1
\end{array}\right] \text {, } \\
& H_{1}=0.1 \text {, } \\
& H_{2}=0.2 \text {. }
\end{aligned}
$$

In addition, the interconnected terms $f_{1}(t, x, p)$ and $f_{2}(t, x, p)$ and the exogenous disturbance terms $\omega_{1}(t)$ and $\omega_{2}(t)$ are selected as

$$
\begin{aligned}
f_{1}(t, x, p) & =0.2 x_{12} x_{22}+0.1 x_{21}+0.2 x_{22} \\
f_{2}(t, x, p) & =0.2 x_{12} x_{22}+0.2 x_{11}+0.2 x_{12} \\
\omega_{1} & =0.2 e^{-t} \cos (3 t) \\
\omega_{2}(t) & =0.1 e^{-3 t} \sin (2 t)
\end{aligned}
$$

Our aim here is to verify the effectiveness of the proposed theoretical results in the previous sections. Combining with the selection of parameters $G_{1}=\left[\begin{array}{ll}0.2 & 0.4\end{array}\right]$ and $G_{2}=\left[\begin{array}{ll}-0.8 & 0.4\end{array}\right]$, and solving LMI condition in Theorem 7, one can obtain that $K_{1}=\left[\begin{array}{lll}1.4552 & 3.6645\end{array}\right]$ and 

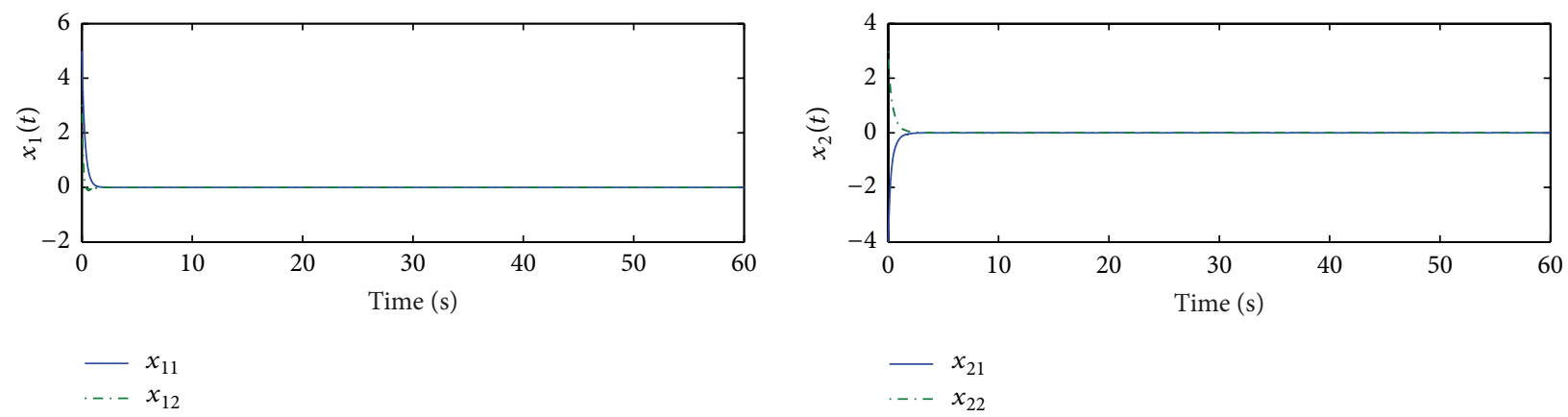

FIGURE 1: The response curves of system states.
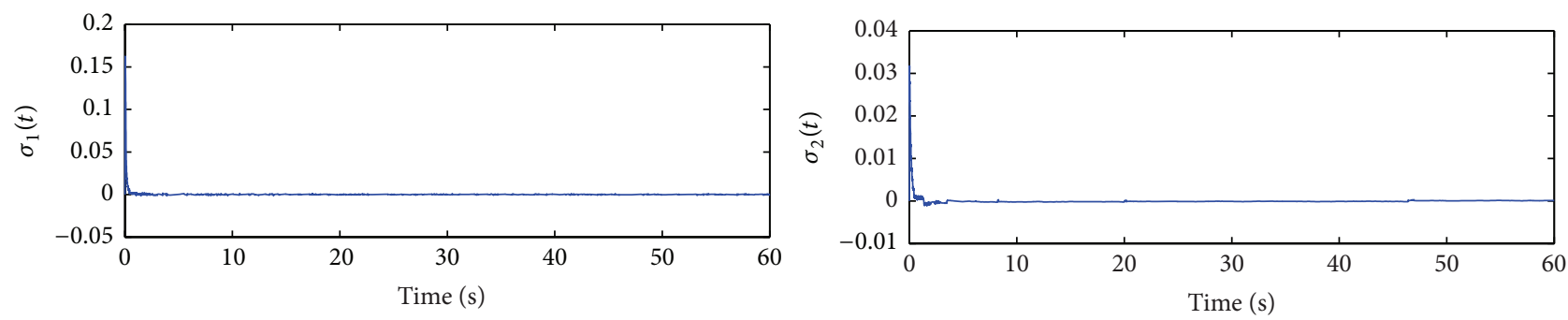

FIGURE 2: The response curves of the switching functions.

$K_{2}=\left[\begin{array}{ll}-6.1215-5.3471\end{array}\right]$. Moreover, the integral-type sliding surface functions in (14) can be computed as

$$
\begin{aligned}
& \sigma_{1}=\left[\begin{array}{ll}
0.2 & 0.4
\end{array}\right] x_{1}-\int_{0}^{t}\left[\begin{array}{ll}
-1.0552 & -2.6645
\end{array}\right] x_{1}(\theta) d \theta \\
& \sigma_{2}=\left[\begin{array}{ll}
-0.8 & 0.4
\end{array}\right] x_{2}-\int_{0}^{t}\left[\begin{array}{ll}
6.9215 & 3.7471
\end{array}\right] x_{2}(\theta) d \theta .
\end{aligned}
$$

In order to illustrate the effect caused by the mismatch of the quantization sensitivity parameters, the evolutions of $\tau_{i c}(t)$ and $\tau_{i d}(t), i=1,2$, are selected to be of the following form:

$$
\begin{aligned}
& \tau_{1 c}(t)= \begin{cases}1.2, & \text { if } t \leq 1, \\
0.8, & \text { if } 1<t \leq 6, \\
0.5, & \text { if } 6<t \leq 15, \\
0.3, & \text { if } t>15,\end{cases} \\
& \tau_{1 d}(t)= \begin{cases}1.4, & \text { if } t \leq 4, \\
0.35, & \text { if } 4<t \leq 12, \\
0.15, & \text { if } 12<t \leq 25, \\
0.068, & \text { if } t>25 .\end{cases} \\
& \tau_{2 c}(t)= \begin{cases}1, & \text { if } t \leq 2, \\
0.6, & \text { if } 2<t \leq 8, \\
0.3, & \text { if } 8<t \leq 20 \\
0.2, & \text { if } t>20\end{cases}
\end{aligned}
$$

$$
\tau_{2 d}(t)= \begin{cases}1.1, & \text { if } t \leq 5 \\ 0.3, & \text { if } 5<t \leq 15 \\ 0.1, & \text { if } 15<t \leq 25 \\ 0.063, & \text { if } t>25\end{cases}
$$

Choosing the parameters $\beta_{1}=2, \beta_{2}=4, \eta_{11}=180, \eta_{21}=$ $300, \eta_{12}=80, \eta_{22}=100, \eta_{13}=20$, and $\eta_{23}=50$ and the initial values of system states $x_{1}(0)=\left[\begin{array}{ll}5 & 3\end{array}\right], x_{2}(0)=\left[\begin{array}{ll}-4 & 3\end{array}\right], \widehat{l}_{1}(0)=$ $0.03, \widehat{l}_{2}(0)=0.02, \widehat{\rho}_{1}(0)=0.001, \widehat{\rho}_{2}(0)=0.006, \widehat{\gamma}_{1}(0)=4$, and $\widehat{\gamma}_{2}(0)=3$, and applying the proposed decentralized adaptive integral sliding mode control strategy proposed in (30)-(33), the simulation results are shown in Figures $1-8$. One can see from Figures 1 and 2 that the proposed decentralized adaptive sliding mode control law can effectively cope with the effect of quantization mismatch, model uncertainties, and external disturbances and guarantee that the system trajectories of the two subsystems can be driven into the desired switching surfaces and convergence to the origin asymptotically. It can be also observed from Figures $3-8$ that $l_{i}, \rho_{i}$, and $r_{i}$ can be well estimated and the estimation error converges into zero in a finite time.

\section{Conclusions}

This paper has been concerned with the problem of decentralized adaptive integral sliding mode control design for a class of nonlinear uncertain large-scale systems subject to quantization mismatch. The LMI conditions are derived for the design of the switching surfaces of each subsystem 

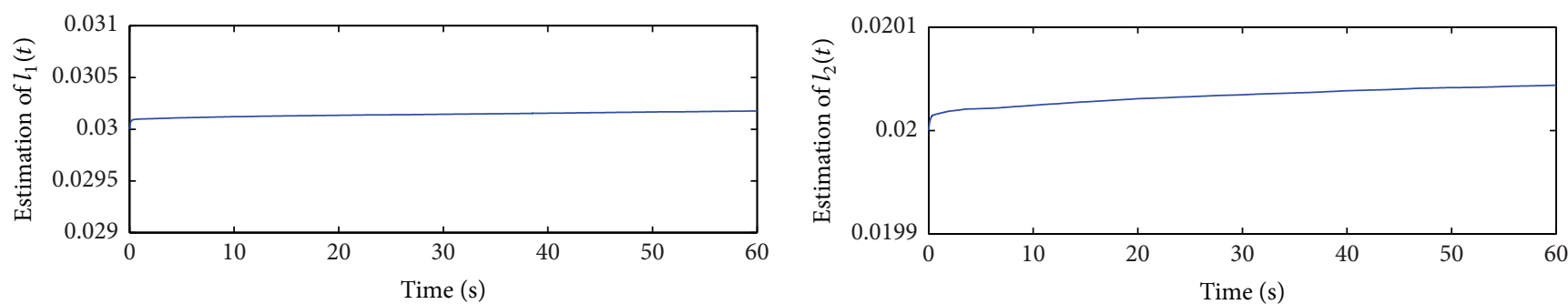

Figure 3: The response curves of $\widehat{l}_{1}$ and $\widehat{l}_{2}$.
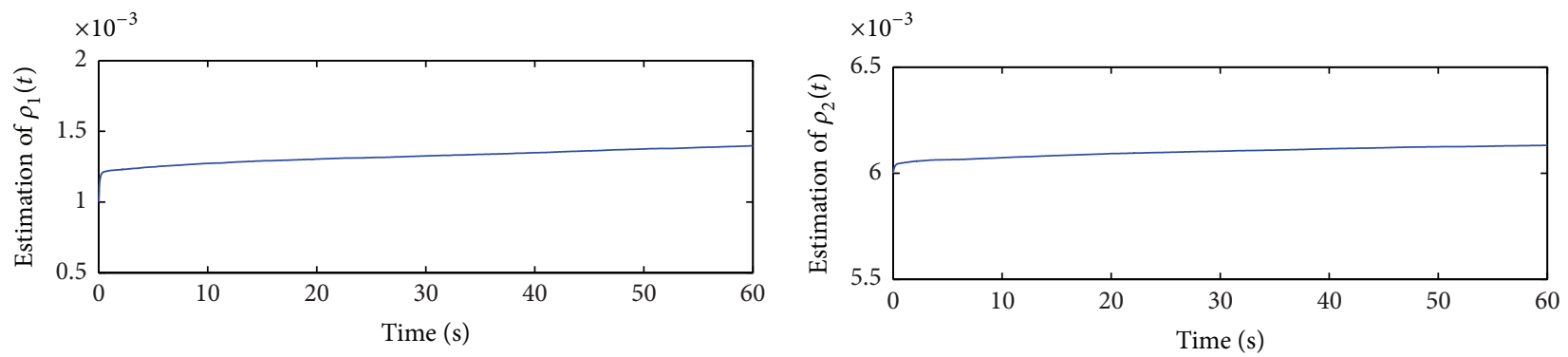

Figure 4: The response curves of $\widehat{\rho}_{1}$ and $\widehat{\rho}_{2}$.
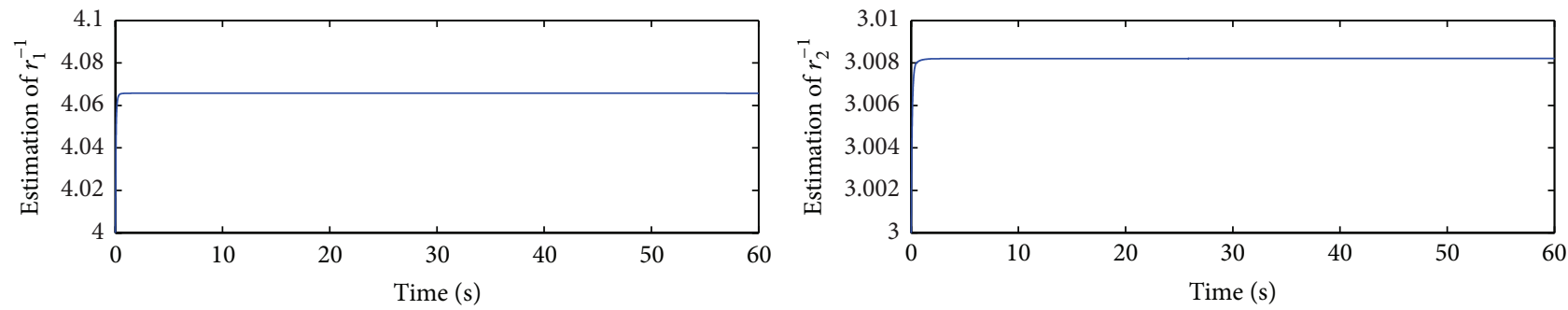

FIgURE 5: The response curves of $\widehat{\gamma}_{1}$ and $\widehat{\gamma}_{2}$.
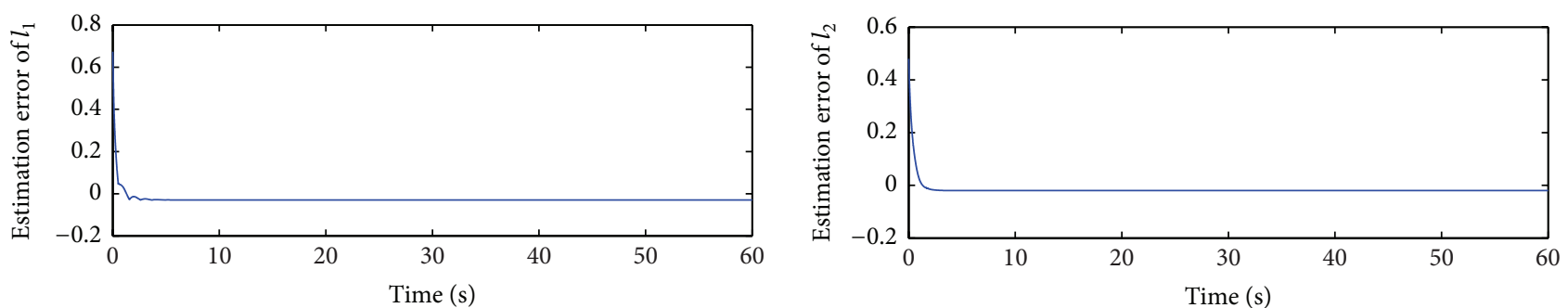

FIGURE 6: The response curves of $\tilde{l}_{1}$ and $\tilde{l}_{2}$.
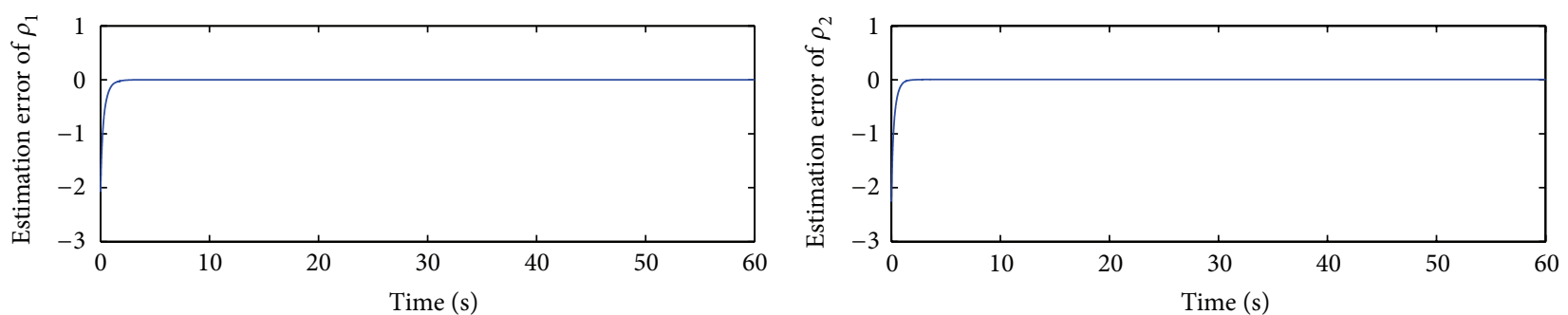

FIGURE 7: The response curves of $\tilde{\rho}_{1}$ and $\tilde{\rho}_{2}$. 

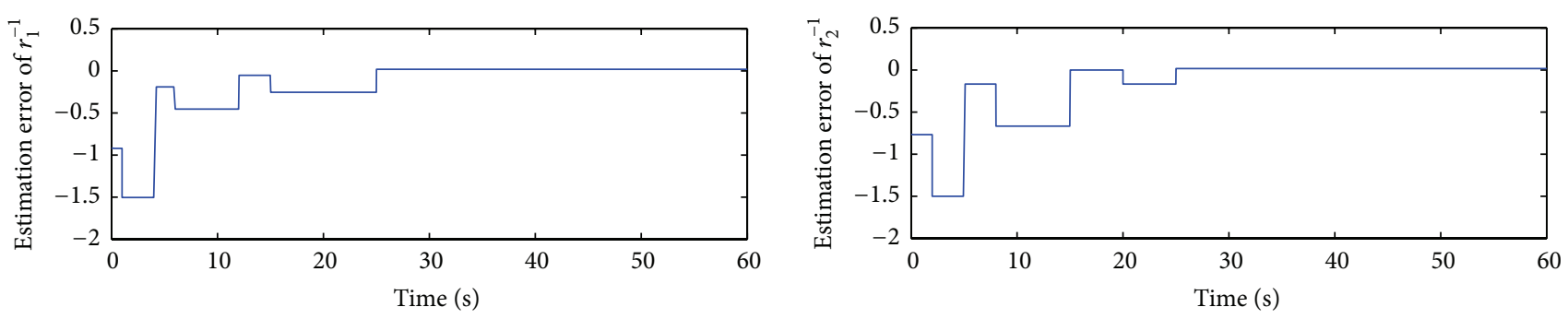

FIGURE 8: The response curves of $\widetilde{\gamma}_{1}$ and $\widetilde{\gamma}_{2}$.

and guaranteeing the bounded $\mathscr{L}_{2}$ gain performance. Furthermore, the decentralized adaptive integral sliding mode control laws are established for ensuring the reachability of the sliding manifold. Finally, an example is given for illustrating the effectiveness of the theoretical results. How to form sliding-mode output feedback control or observedbased sliding mode control for solving the more kinds of disturbances, including the constant $w(t)$ as special case, will be an important and interesting research topic in our future work.

\section{Conflict of Interests}

The authors declare that there is no conflict of interests regarding the publication of this paper.

\section{Acknowledgments}

This work was supported in part by the National Natural Science Foundation of China (Grants nos. 11301276, 61403207, and 61273155) and by the Natural Science Foundation of Jiangsu Province (BK20130984 and BK20131000).

\section{References}

[1] R. W. Brockett and D. Liberzon, "Quantized feedback stabilization of linear systems," IEEE Transactions on Automatic Control, vol. 45, no. 7, pp. 1279-1289, 2000.

[2] D. Liberzon, "Hybrid feedback stabilization of systems with quantized signals," Automatica, vol. 39, no. 9, pp. 1543-1554, 2003.

[3] T. Li and L. Xie, "Distributed coordination of multi-agent systems with quantized-observer based encoding-decoding," IEEE Transactions on Automatic Control, vol. 57, no. 12, pp. 3023-3037, 2012.

[4] S. Hu and D. Yue, "Event-triggered control design of linear networked systems with quantizations," ISA Transactions, vol. 51, no. 1, pp. 153-162, 2012.

[5] L. Li, Y. Xia, J. Qiu, and H. Yang, "Robust $H_{\infty}$ networked control for discrete-time fuzzy systems with state quantisation," International Journal of Systems Science, vol. 43, no. 12, pp. 22492260, 2012.

[6] L. Zhou and G. Lu, "Quantized feedback stabilization for networked control systems with nonlinear perturbation," International Journal of Innovative Computing, Information and Control, vol. 6, no. 6, pp. 2485-2495, 2010.
[7] W.-W. Che and G.-H. Yang, " $H_{\infty}$ filter design for continuoustime systems with quantised signals," International Journal of Systems Science, vol. 44, no. 2, pp. 265-274, 2013.

[8] M. L. Corradini and G. Orlando, "Robust quantized feedback stabilization of linear systems," Automatica, vol. 44, no. 9, pp. 2458-2462, 2008.

[9] B.-C. Zheng, G.-H. Yang, and T. Li, "Quantised feedback sliding mode control of linear uncertain systems," IET Control Theory \& Applications, vol. 8, no. 7, pp. 479-487, 2014.

[10] L.-Y. Hao and G.-H. Yang, "Fault-tolerant control via slidingmode output feedback for uncertain linear systems with quantisation," IET Control Theory \& Applications, vol. 7, no. 16, pp. 1992-2006, 2013.

[11] Y. Yan and X. Yu, "Quantization behaviors in equivalent-control based sliding-mode control systems," in Advances in Sliding Mode Control, B. Bandyopad-hyay, S. Janardhanan, and S. Spurgeon, Eds., pp. 221-239, Springer, London, UK, 2013.

[12] M. Liu, X. Cao, S. Zhang, and W. Yang, "Sliding mode control of quantized systems against bounded disturbances," Information Sciences, vol. 274, pp. 261-272, 2014.

[13] S. W. Yun, Y. J. Choi, and P. Park, " $H_{2}$ control of continuous-time uncertain linear systems with input quantization and matched disturbances," Automatica, vol. 45, no. 10, pp. 2435-2439, 2009.

[14] Y. Zou, Y. Niu, B. Chen, and T. Jia, "Networked predictive control of constrained linear systems with input quantisation," International Journal of Systems Science, vol. 44, no. 10, pp. 19701982, 2013.

[15] J. Zhou, C. Wen, and G. Yang, "Adaptive backstepping stabilization of nonlinear uncertain systems with quantized input signal," IEEE Transactions on Automatic Control, vol. 59, no. 2, pp. 460-464, 2014.

[16] M. Fu and L. Xie, "The sector bound approach to quantized feedback control," IEEE Transactions on Automatic Control, vol. 50, no. 11, pp. 1698-1711, 2005.

[17] T. Kameneva and D. Nešić, "Robustness of quantized control systems with mismatch between coder/decoder initializations," Automatica, vol. 45, no. 3, pp. 817-822, 2009.

[18] T. Kameneva and D. Nešić, "Robustness of nonlinear control systems with quantized feedback," Nonlinear Analysis: Hybrid Systems, vol. 4, no. 2, pp. 306-318, 2010.

[19] C. D. Persis, "Robustness of quantized continuous-time nonlinear systems to encoder/decoder mismatch," in Proceedings of the 48th IEEE Conference on Decision and Control, pp. 13-18, Shanghai, China, December 2009.

[20] B.-C. Zheng and G.-H. Yang, " $H_{2}$ control of linear uncertain systems considering input quantization with encoder/decoder mismatch," ISA Transactions, vol. 52, no. 5, pp. 577-582, 2013.

[21] X.-G. Yan, J.-J. Wang, X.-Y. Lü, and S.-Y. Zhang, “Decentralized output feedback robust stabilization for a class of nonlinear 
interconnected systems with similarity," IEEE Transactions on Automatic Control, vol. 43, no. 2, pp. 294-299, 1998.

[22] X.-G. Yan, C. Edwards, and S. K. Spurgeon, "Decentralised robust sliding mode control for a class of nonlinear interconnected systems by static output feedback," Automatica, vol. 40, no. 4, pp. 613-620, 2004.

[23] H.-T. Yau and J.-J. Yan, "Robust decentralized adaptive control for uncertain large-scale delayed systems with input nonlinearities," Chaos, Solitons and Fractals, vol. 39, no. 4, pp. 1515-1521, 2009.

[24] M. S. Mahmoud, A. Y. Al-Rayyah, and Y. Xia, "Quantised feedback stabilisation of interconnected discrete-delay systems," IET Control Theory \& Applications, vol. 5, no. 6, pp. 795-802, 2011.

[25] B.-C. Zheng and G.-H. Yang, "Decentralized sliding mode quantized feedback control for a class of uncertain large-scale systems with dead-zone input," Nonlinear Dynamics, vol. 71, no. 3, pp. 417-427, 2013.

[26] L. Wu, X. Su, and P. Shi, "Sliding mode control with bounded $\mathscr{L}_{2}$ gain performance of Markovian jump singular time-delay systems," Automatica, vol. 48, no. 8, pp. 1929-1933, 2012.

[27] I. R. Petersen, "A stabilization algorithm for a class of uncertain linear systems," Systems \& Control Letters, vol. 8, no. 4, pp. 351357, 1987.

[28] F. Castaños, D. Hernández, and L. M. Fridman, "Integral sliding-mode control for linear time-invariant implicit systems," Automatica, vol. 50, no. 3, pp. 971-975, 2014.

[29] Y. Niu, D. W. C. Ho, and J. Lam, "Robust integral sliding mode control for uncertain stochastic systems with time-varying delay," Automatica, vol. 41, no. 5, pp. 873-880, 2005.

[30] C. Edwards and S. K. Spurgeon, Sliding Mode Control: Theory And Applications, Taylor \& Francis, London, UK, 1998.

[31] V. I. Utkin, Sliding Modes in Control and Optimization, Communications and Control Engineering Series, Springer, Berlin, Germany, 1992.

[32] Q. Hu, G. Ma, and L. Xie, "Robust and adaptive variable structure output feedback control of uncertain systems with input nonlinearity," Automatica, vol. 44, no. 2, pp. 552-559, 2008.

[33] F. H. Clarke, Y. S. Ledyaev, E. D. Sontag, and A. I. Subbotin, "Asymptotic controllability implies feedback stabilization," IEEE Transactions on Automatic Control, vol. 42, no. 10, pp. 1394-1407, 1997.

[34] M. Krstić and P. V. Kokotović, "Control Lyapunov functions for adaptive non-linear stabilization," Systems and Control Letters, vol. 26, no. 1, pp. 17-23, 1995.

[35] Y. S. Ledyaev and E. D. Sontag, "A Lyapunov characterization of robust stabilization," Nonlinear Analysis: Theory, Methods \& Applications, vol. 37, no. 7, pp. 813-840, 1999. 


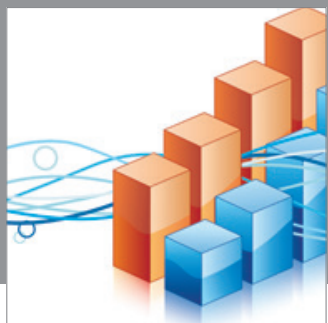

Advances in

Operations Research

mansans

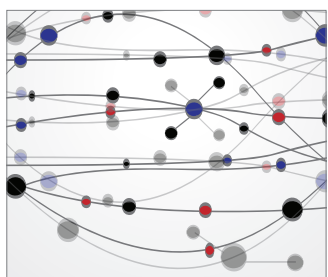

The Scientific World Journal
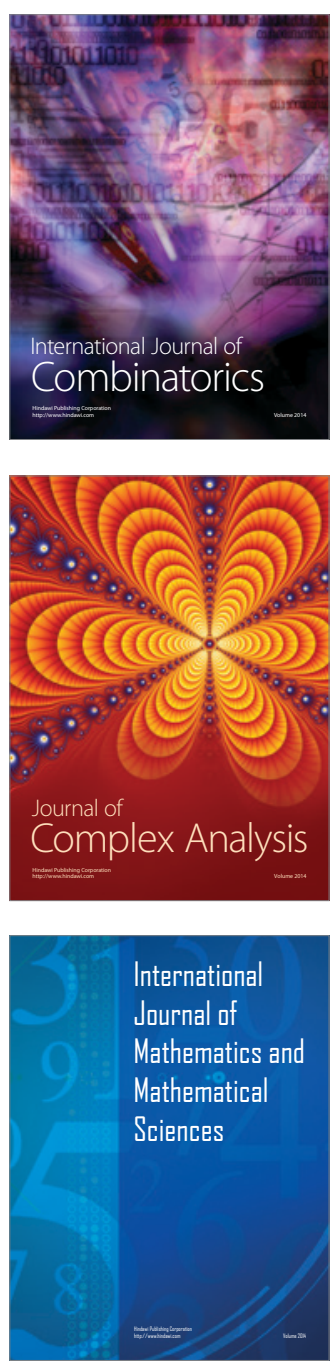
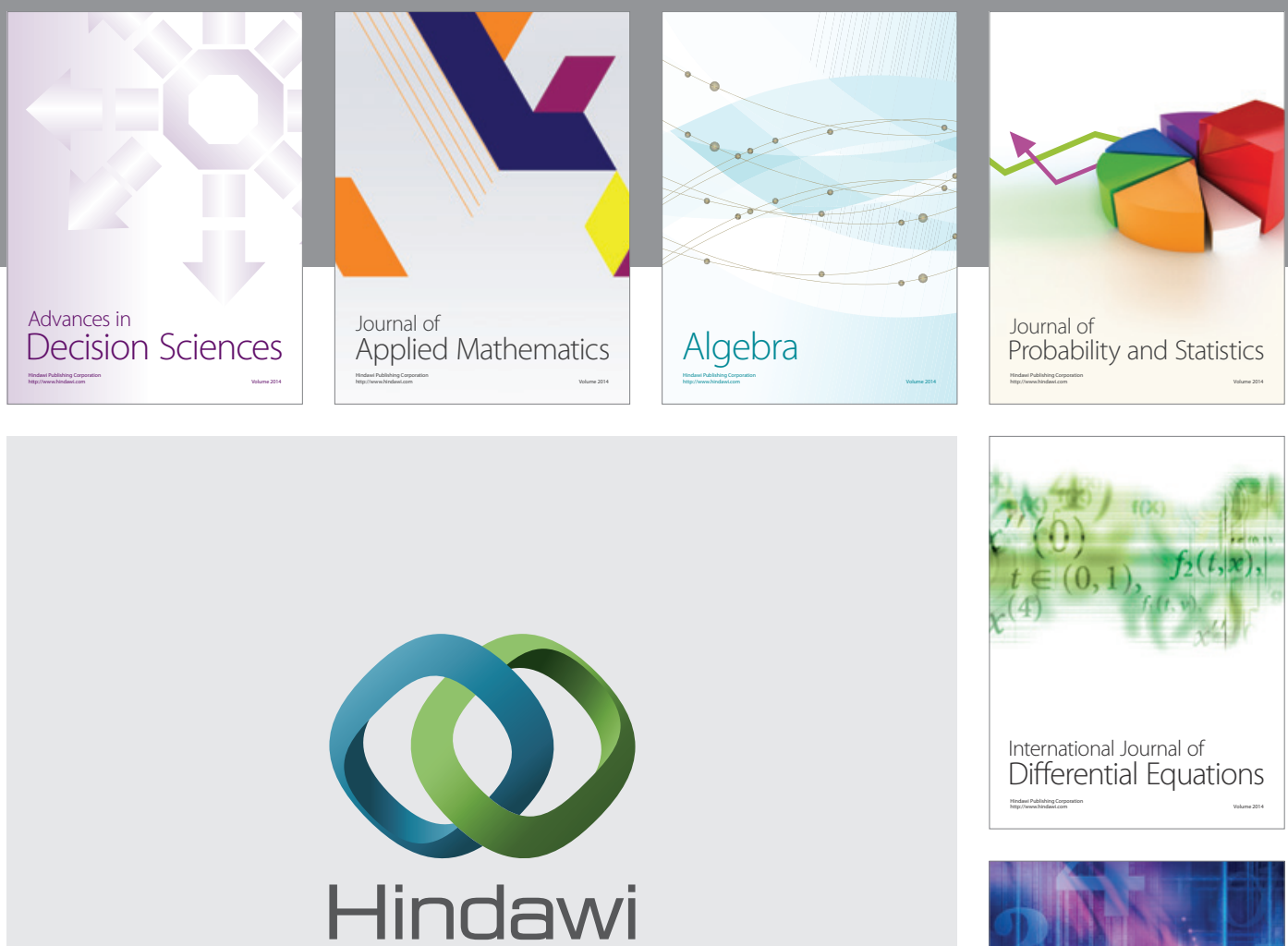

Submit your manuscripts at http://www.hindawi.com
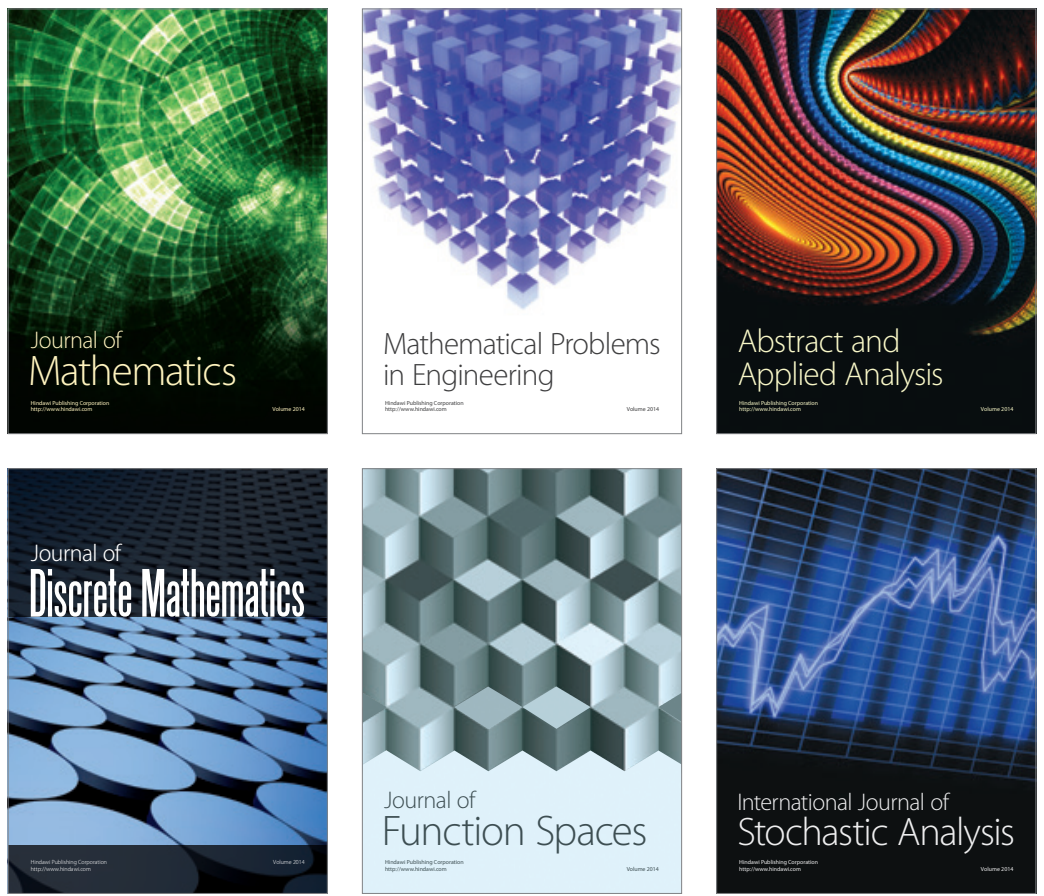

Journal of

Function Spaces

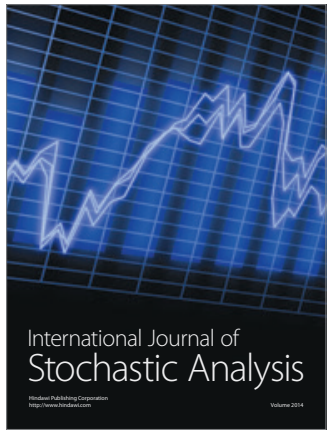

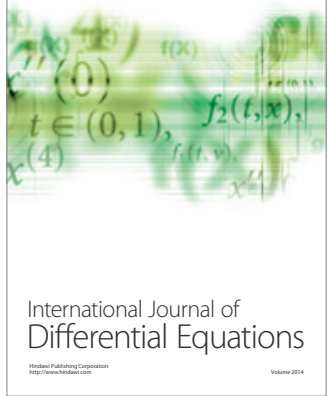
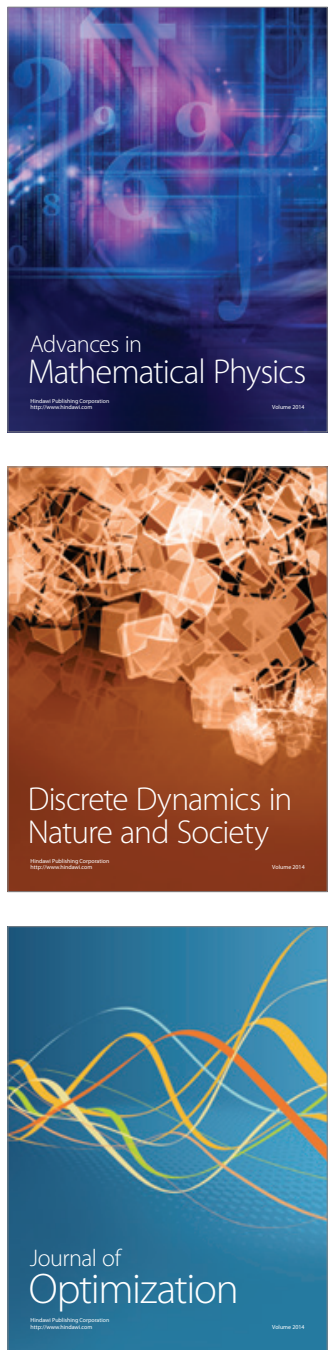